\title{
Les mutations des mondes et du syndicalisme portuaires autour de 1968.
}

\author{
Michel Pigenet \\ Centre d'histoire sociale du XXe siècle, (Université Paris 1/CNRS))
}

« De 1968, les dockers sortent unis et forts. Et c'est à ce moment là que le déclin commence (...). La modernisation galope. Les ports, les bateaux, les transports, la manutention, la concurrence, tout va changer, très vite. Et nous on va faire avec. Moi comme les autres. ${ }^{1}$ Il serait commode de lire dans ce constat, un rien désabusé, dressé par un ancien docker marseillais, le constat nostalgique des déconvenues éprouvées en une fin de XXe siècle plus subie que maîtrisée. Pour ne pas être fausse, cette lecture ne saurait masquer l'expression, non moins remarquable, des paradoxes, ambivalences et contradictions de 1968. Sans doute la situation dans les ports mérite-t-elle de retenir l'attention par les exagérations et exacerbations qu'elle présente et que l'on se propose d'aborder du triple point de vue des événements, des mutations engagées au moment où ils se déroulent et de celles qu'ils précipitent.

\section{Les images brouillées du 68 portuaire}

\section{La suprématie cégétiste à la veille des événements}

En 1966, une enquête diligentée par l'administration évalue à 94,7 \% l'influence cégétiste parmi les quelques 16000 dockers professionnels, quand Force ouvrière et les Indépendants doivent se contenter de $2,6 \%$ chacun. $^{2}$ FO conteste ces chiffres, mais la composition des conseils d'administration paritaires des bureaux centraux de main-d'œuvre BCMO -, pourtant désignés par l'administration, témoigne de l'audience de la CGT qui monopolise la représentation salariale dans vingt-et-un des vingt-sept ports concernés. ${ }^{3}$

Cette suprématie ancienne, écornée à la suite des rudes conflits de guerre froide du début des années $1950^{4}$, se renforce au cours de la décennie suivante. Cela vaut en premier lieu pour Marseille où le syndicat des dockers, imprudemment lancé en 1950 dans une longue grève, subit une défaite qui entraîna son effondrement durable. Il faut attendre 1961 pour voir l'organisation refaire surface et reprend l'initiative. Depuis cette date, ses militants reconstituent méthodiquement leurs rangs et obligent leurs rivaux à se déterminer par rapport à leurs revendications et modes d'action. En février 1966, le syndicat des dockers annonce 500 membres auxquels il convient d'ajouter 700 adhérents parmi les dockers mensualisés, spécialistes et agents de maîtrise, davantage épargnés par la tourmente de $1950 .^{5}$

A l'échelon national la CGT recueille également le soutien de 70 à $80 \%$ des personnels techniques des ports autonomes et des chambres de commerce affectés à la

\footnotetext{
${ }^{1}$ A. Pacini/D. Pons, Dockers à Marseille, Paris, Payot, 1996, p. 217.

${ }^{2}$ Note du Service central au Directeur des ports maritimes et des voies navigables (pmvn), le 3 mars 1966. Centre des archives contemporaines (CAC), 870150 art. 113.

${ }^{3}$ Idem. A Bordeaux, Dieppe, Marseille, Nantes, Port-La Nouvelle, Saint-Nazaire et Sète, elle siège au côté de syndicalistes FO, Autonomes ou Indépendants.

${ }^{4}$ M. Pigenet, «Les dockers français, 'gardes-côtes de la paix' », in Images et mouvements du siècle. Chronique sociale, t. 2, Les raisons de la colère, Institut CGT d'Histoire sociale-Editions France Progrès, 1999, p. 409-416. ${ }^{5}$ Lettre de Désiré Brest, secrétaire général de la Fédération CGT des Ports et Docks au Directeur des pmvn, le 4 février 1966, CAC, 870150, art. 113.
} 
conduite des grues et autres outillages publics. Son influence est également importante parmi les marins, les officiers et les officiers-mécaniciens de la navigation commerciale.

Acteurs indispensables des ruptures de charges et des transbordements portuaires des marchandises, les dockers ont conscience d'occuper une position stratégique dans la chaîne des transports. L'organisation aidant, ce pouvoir d'influence explique une conflictualité élevée et diversifiée. Au gré des revendications et des circonstances, ils excellent dans l'articulation de la paralysie générale des quais pour 24 à 48 heures aux grèves perlées, à la limitation des rendements, à la suspension des heures supplémentaires, du travail de nuit ou dominical, sources de coûteuses surestaries pour les navires immobilisés. A quoi le patronat réplique de temps à autre par des lock-out. Aussi rugueuses qu'y soient les relations sociales, les ports sont simultanément des hauts lieux de la négociation collective. L'épaisseur et la minutie des conventions locales rendent compte du sérieux des discussions menées selon des procédures rôdées au sein d'instances pérennes. La loi du 6 septembre 1947 fixe la composition et les prérogatives des BCMO paritaires qui supervisent, sous la présidence des directeurs des ports, l'attribution des cartes professionnelles et l'embauche biquotidienne des dockers intermittents.

\section{8 dans les ports}

Les travailleurs portuaires répondent massivement au mot d'ordre de grève interprofessionnelle lancé pour le 13 mai. Leur présence ne passe pas inaperçue dans les cortèges des villes littorales, mais les foules rassemblées relativisent cette fois leur participation. Le 14, la reprise s'accompagne, tout au long de la semaine, d'une recrudescence d'escarmouches à base de refus des heures supplémentaires et du travail de nuit. Hormis Rouen où la proximité de Cléon précipite, peut-être, le déclenchement de la grève le 17 , celleci ne démarre vraiment ailleurs qu'à partir du 20.

Praticiens expérimentés de l'arrêt de travail, les dockers se méfient des mouvements précipités et désordonnés. Les dirigeants de la Fédération CGT des Ports et Docks entretiennent la mémoire des expériences cuisantes de 1950 et, plus précisément, des erreurs commises à Marseille, dont les militants étaient passés outre les consignes nationales de prudence. La circulaire fédérale du 17 mai propose ainsi une interprétation minimaliste de l'orientation adoptée le même jour par le comité confédéral national de la CGT. Quand ce dernier préconise, "pour le moment », d' " étendre la grève par en bas », les cégétistes des Ports et Docks estiment qu'il ne serait «pas efficace d'avoir recours à la grève générale illimitée ${ }^{6}{ }^{6}$ Surtout, recommandent-ils à l'adresse de leurs camarades, «n'abandonnez pas le chantier ni le port ». Le conseil vise à contrecarrer une éventuelle déclaration de «l'embauche libre », mesure administrative suspensive de la priorité reconnue aux dockers professionnels. Des militants souligneront après coup la circonspection qui prévaut alors. " Nous avions mal compris comment le mouvement était parti (..), mal compris la position de la CGT», confiera le secrétaire du syndicat des grutiers du port autonome de Nantes-Saint-Nazaire. ${ }^{7}$

Tout change, à compter du 20 mai, avec le blocage de l'ensemble des ports, lequel précipite celui du pays. Afin de garder la maîtrise des sites, les dockers optent pour l'occupation. L'affaire ne va pas de soi au regard des vastes espaces à surveiller, des entrepôts et des marchandises à protéger contre les risques de dégradations ou de vols. Soumis au contrôle des assemblées générales, les cégétistes se retrouvent quasiment seuls aux postes de commande du mouvement. ${ }^{8}$ Rompus aux exigences de coordination et de discipline, ceux du

\footnotetext{
${ }^{6}$ Circulaire citée par Laffitte, Congrès fédéral national, Paris, 20-21 juin 1968, Imprimerie de la FNPD, p. 32.

${ }^{7}$ Intervention de Dubourg, Idem, p. 22.

${ }^{8}$ A Marseille, la CGT accepte une certaine unité d'action avec la CFDT, très minoritaire et traversée de courants contradictoires.
} 
Havre réussissent à affecter un dixième des travailleurs à la tenue des piquets qui stationnent devant les entrées du port, tandis que près de quatre cents voitures particulières patrouillent à l'intérieur de l'enceinte. ${ }^{9}$ Ici, comme à Rouen, Dunkerque ou Bordeaux, le port donne son ton à la grève locale. A Marseille, les 4000 dockers pèsent lourd lors des manifestations et dans le rapport des forces sociales et syndicales. ${ }^{10}$ On devine ce qu'il en est dans les villes plus modestes telles que Sète, Bayonne, Lorient, Dieppe ou La Rochelle.

Il en va autrement à l'échelon national où l'ampleur de la grève dilue la contribution spécifique des «portuaires » ignorés par la plupart des travaux consacrés à 1968. ${ }^{11}$ Le défaut de curiosité s'étend aux modalités d'une reprise difficile. Un premier accord intervenu le 4 juin ne concerne que les personnels des ports autonomes et des chambres de commerce. Pour les dockers, la conclusion des négociations, commencées ce même jour au siège de l'Union nationale des industries de la manutention - UNIM - ${ }^{12}$ tarde à venir. Quatre jours ne seront pas de trop entre l'établissement, au petit matin du 6, d'un constat d'accord et sa signature, le 10. D'un bout à l'autre, la Fédération CGT veille à préserver sa position d'interlocuteur privilégié du patronat. Ses représentants refusent ainsi de discuter en présence des autres organisations syndicales. ${ }^{13}$ Au vrai, les employeurs savent à quoi s'en tenir : «C'est vous qui faites la discussion », reconnaît le Président de l'UNIM devant la délégation cégétiste. ${ }^{14}$

\section{Une sortie de grève difficile}

Sans être négligeable, le contenu de l'«accord Marceau »n'a rien d'exceptionnel. Fixée à $20 \%$, la hausse, étalée sur six mois, du salaire de base est inférieure à celle du SMIG auquel il était jusque-là indexé. Il enregistre néanmoins une progression supérieure aux moyennes de Grenelle. L'augmentation atteint $10 \%$ pour les primes de rendement et l'on programme de prochaines rencontres sur la réduction de la durée du travail. La Caisse nationale de garantie des ouvriers dockers - Cainagod -, l'organisme paritaire alimenté par des cotisations patronales de financement de l'indemnisation des vacations chômées des dockers professionnels, s'engage à assurer le paiement des jours de grève. ${ }^{15}$ La principale cause d'étonnement réside moins dans les résultats que de l'expression devant les employeurs des dissensions cégétistes au cours des négociations. Jugé trop conciliant et oublieux des ultimes positions fédérales arrêtées le $5,{ }^{16}$ le secrétaire général de la Fédération, Désiré Brest, est à plusieurs reprises contredit, voire interrompu, par ses camarades. Les délégués du Havre, de Rouen et de Bordeaux marquent leurs désaccords à l'instar des Marseillais et des Dunkerquois, opposants de plus longue date en dépit d'une appartenance partagée au PCF. L'un d'eux songe même à claquer la porte avant de se raviser. ${ }^{17}$ Tous reprochent au chef de délégation de s'en tenir au relevé de conclusions de Grenelle et, plus encore, d'avoir déclaré, au terme d'une nuit passée à débattre des salaires, qu'il était «trop tard » pour traiter de la réduction du temps de travail.

\footnotetext{
${ }^{9}$ Intervention de Nolent, Congrès fédéral national, Paris, 20-21 juin 1968, op. cit., p. 39.

${ }^{10}$ Poids plus marqué encore si l'on considère les 20000 salariés de la zone portuaire.

${ }^{11}$ Y compris par celui de X. Vigna, L'insubordination ouvrière dans les années 1968. Essai d'histoire politique des usines, Rennes, PUR, 2007, pourtant le plus attentif aux luttes ouvrières.

1276 avenue Marceau, à Paris.

${ }^{13}$ Ils se retirent après avoir entrevu des rivaux auxquels ils dénient toute représentativité dans les locaux de l'UNIM. Interventions d'Henry et de Gabas, Congrès fédéral national, Paris, 20-21 juin 1968, op. cit., p. 72 et 82.

${ }^{14}$ Idem.

${ }^{15}$ L'accord comporte d'autres clauses, dont l'institution d'un huitième jour férié.

${ }^{16}$ Le 5 juin, la délégation cale ses positions et décide de tenir ferme sur la réduction de la durée du travail de 30 minutes par vacation ou une heure par jour ainsi que sur la réévaluation de l'indemnité de garantie pour les vacations chômées.

${ }^{17}$ Intervention de Brachet, Congrès fédéral national, Paris, 20-21 juin 1968, op. cit., p. 47.
} 
La disparité des situations locales, où des négociations se déroulent parallèlement, aggrave et éclaire la confusion parisienne. Si la plupart des assemblées générales se prononcent en faveur du compromis réalisé, les dockers de Marseille et de Dunkerque, convaincus de pouvoir arracher de meilleurs résultats, souhaitent retarder la date de la signature nationale, signal d'une reprise générale qui les mettrait à découvert. Sur place, les employeurs se prévalent d'ailleurs de l'accord du 10 juin pour discréditer les prolongations. A Marseille, un tract patronal, tiré à 100000 exemplaires, dénonce «l'intransigeance » du syndicat phocéen opposé au réalisme fédéral. Après d'ultimes péripéties, les négociations finissent par aboutir les 17 et 19 juin, à Dunkerque et Marseille. A la Joliette, l'épreuve de forces achève de restaurer l'autorité de la CGT. Outre la préservation des avances salariales marseillaises, l'établissement d'une priorité spécifique d'emploi pour les dockers âgés et l'attribution de nouvelles cartes professionnelles, les cégétistes obtiennent la moitié des postes de contrôleurs d'embauche et un siège supplémentaire au conseil d'administration du BCMO.

\section{Le congrès orageux des 20 et 21 juin : premières retombées fédérales du mouvement}

Le 20 juin, quelques heures après la reprise marseillaise, les 143 délégués au congrès fédéral se retrouvent à Paris pour deux jours de débats dont chacun sait qu'ils seront orageux. La direction n'a pas voulu repousser la date des assises fixée avant les événements. Plusieurs militants lui reprochent une obstination qui sent la manœuvre. Si le soupçon n'est pas infondé, le défaut de discussion sur un rapport d'activité obsolète se retourne contre ses auteurs, pris à contre-pied par une grève historique. Le vieillissement brutal des références accuse l'âge des responsables, au premier chef, du secrétaire général. A 69 ans, l'ancien mutin de 1919, batelier « unitaire » de l'entre-deux-guerres, dirigeant fédéral permanent controversé de 1944 et secrétaire général depuis 1962, a connu d'autres congrès houleux au sein d'une organisation composite, riche en fortes personnalités irréductibles à leurs attaches partisanes. Cette fois, pourtant, la contestation déborde de son périmètre traditionnel. Ebranlés, les soutiens habituels de Désiré Brest se font discrets. Le rejet majoritaire du rapport d'activité n'annonce rien de bon pour le vieux dirigeant qui prend les devants pour déclarer qu'il renonce à son poste et accepte d'accéder à une présidence honoraire, « ce qui se fait quand les gens quittent dans l'honneur et la gloire ${ }^{18}$ Son remplacement par Gaston Henry, un docker parisien de vingt ans plus jeune que son lui, s'accompagne d'un net rajeunissement des instances nationales. La moyenne d'âge du bureau fédéral s'abaisse à 43 ans et 9 mois, soit 7 ans de moins que son prédécesseur élu en 1966. La commission exécutive est elle-même largement reconstituée par l'entrée de sept nouveaux membres parmi des treize qui la composent.

Au total, 1968 fait office, dans les ports aussi, de révélateur et d'accélérateur. Le congrès qui suit la grève prend acte du tournant opéré. Parties prenantes d'un mouvement général dont on ne saurait les isoler, les grèves de dockers gardent néanmoins dans leur déroulement, conclusions et retombées, des particularités qui renvoient à des enjeux et dynamiques spécifiques.

\section{8 dans le processus des mutations portuaires des années 1960-1970}

\section{Les ports au miroir des évolutions de la société française}

\footnotetext{
${ }^{18}$ Intervention de Brest, Idem, p. 80. Désiré Brest crut un moment qu'il continuerait à garder la haute main sur la Fédération ce qui nécessita une douloureuse mise au point et entraîna un détachement progressif d'avec ses anciens camarades qui l'élimineront de la prochaine commission exécutive.
} 
La fermeture relative de la profession ne coupe pas les dockers du gros des travailleurs dont ils partagent les conditions d'existence, les quartiers de résidence et la culture. L'ancrage revendiqué, intermittence oblige, dans un passé antérieur aux normes salariales keynesofordistes les prédispose toutefois moins que d'autres à adopter les nouveaux modes de vie consécutifs à l'amélioration du pouvoir d'achat ouvrier. Dans un milieu vieillissant, ${ }^{19}$ les malentendus générationnels empruntent volontiers à la rhétorique de classe. En 1962, un syndicaliste bordelais s'émeut de ce que les «jeunes oublient le travail » et s'absentent sans raison pour «pomper au chômage ». ${ }^{20}$ «Nous ne sommes pas du tout contents de nos camarades » qui voudraient ainsi travailler à terre - jadis un «déshonneur » - plutôt que sur les navires, afin de s'éclipser pour aller au cinéma, chez le coiffeur ou festoyer entre amis. Solidaires dans l'indiscipline - «les jeunes dockers se tiennent les coudes »-, mais indéfendables, ils mettraient le syndicat « dans une position épouvantable ».

Conscient de l'irréversibles des changements intervenus, un autre « ancien » convoque ses souvenirs pour appeler ses camarades à plus de compréhension. "Autrefois, explique-t-il, un bleu de travail, des charentaises nous suffisaient. Quand nous étions jeunes, nous pouvions nous permettre, le samedi, d'engueuler la patronne du garni ou du meublé si nous n'avions pas assez de travail et ne pouvions pas payer. Aujourd'hui, c'est différent avec les HLM. Si elles ne sont pas payées, on n'a plus qu'à faire la valise ». ${ }^{21}$ Les jeunes « ont des besoins différents de ceux que nous avions », insiste-t-il avant de citer l'automobile, la télévision, le réfrigérateur, la machine à laver... Le syndicaliste va plus loin. Dans cinq à dix ans, prévient-il à l'adresse des militants tentés de faire «l'autruche», ces aspirations poseront en termes inédits le problème de la «permanentisation ».

La question n'est pas de celle que l'on soulève à la légère sur les quais, domaine d'une intermittence dissociée de la précarité depuis 1947. Entre vigilance et crispation, la Fédération redoute les effets d'un recrutement laxiste et s'alarme des conceptions dissolvantes de «jeunes dockers venant de professions voisines [qui] veulent travailler six jours la semaine comme en usine... ${ }^{22}$ Tous âges confondus, les plus pessimistes se désolent de ce que la nouvelle génération n'ait pas «l'esprit docker», ne sache «pas se battre » et «démolisse la profession $»{ }^{23}$ A quoi certains répliquent que mieux vaudrait « commencer à faire confiance aux jeunes ». Exemple de Dunkerque à l'appui, l'un d'eux montre comment l'élection d'un bureau syndical dont la moyenne d'âges avoisine 30 ans a entraîné un regain de combativité sur le modèle, prémonitoire en 1962, de «brigades de jeunes qui « aimeraient retrouver ce dont les vieux nous ont parlé : occupation du port, piquets de grève... ${ }^{24}{ }^{24}$

Ces tensions n'échappent aux employeurs et aux pouvoirs publics. « Les dockers sont attachés au système actuel qui leur garantit beaucoup de liberté », note un proche de ChabanDelmas. ${ }^{25}$ «Il n'est pas sûr, ajoute-t-il, que les jeunes générations voient les choses de cette manière ».

\footnotetext{
${ }^{19}$ Une étude administrative établit à 44 ans et 5 mois, l'âge moyen de l'ensemble des dockers au $1^{\text {er }}$ janvier 1970. CAC 870150, art. 161, Statistiques du Service central de la direction des pmvn, le 17 novembre 1970.

${ }^{20}$ Intervention de Frédou, Congrès fédéral national, Paris, 13-14 juin 1962, p. 18. Alfred Breillac, dit Frédou, dirigea le syndicat de Bordeaux de 1958 à 1966.

${ }^{21}$ Intervention de Vaubaillon, Idem, p. 30. Louis Vaubaillon, alors âgé de 53 ans, dirige le syndicat de Rouen. Membre du bureau fédéral depuis 1957, il fut secrétaire fédéral de 1962 à 1968.

${ }^{22}$ L'Avenir des Ports, juin 1967.

${ }^{23}$ Intervention de Nolent, Congrès fédéral national, Paris, 17-18 juin 1964, p. 64. A 35 ans, Lucien Nolent dirige depuis deux ans le puissant syndicat du Havre.

24 Intervention de Gouvart, Congrès fédéral national, Paris, 13-14 juin 1962, p. 71-72. Rajeunissement qu'illustre Roger Gouvart, âgé de 34 ans en 1962 et membre de la direction de son syndicat depuis 1958.

${ }^{25}$ Note relative au conflit des Ports et Docks d'Yves Chaigneau, chargé de mission auprès du Premier Ministre, le 16 décembre 1970. CAC, 870150, art. 165.
} 
A l'aube des années 1960, l'augmentation de la taille des navires marchands et leur spécialisation restreint le nombre des ports d'accueil et relance leur compétition. Il s'agit toujours de réduire les périodes d'immobilisation et de disposer au meilleur prix des hommes et des équipements nécessaires. L'intermittence des ouvriers et l'utilisation d'engins loués sur place ont longtemps suffit. ${ }^{26} \mathrm{La}$ mécanisation tardive, mais accélérée des manutentions concourt à modifier la donne. En usage dès les années 1940, les chariots-élévateurs se généralisent. Les années 1960 sont aussi celles de l'arrivée des conteneurs. Ceux-ci transforment radicalement les opérations de manutention découplées du rempotage-dépotage des marchandises effectuées à distance des enceintes publiques réservées aux dockers professionnels. Les perspectives de délocalisation d'une partie du travail se superposent à celles attendues de la rationalisation qu'autorisent les grandes boîtes métalliques. Plus rapide, le chargement et le déchargement des bateaux réclame des hommes aptes à conduire les engins dont le coût élimine les petites entreprises et pousse au recrutement de salariés «permanents ». En rupture, donc, avec la tradition de l'intermittence et les règles fixées en 1947..$^{27} \mathrm{Au}$ même moment, l'industrialisation des littoraux pose les questions explosives de l'usage privatif du domaine public par les usines construites « au bord de l'eau » et du personnel habilité à s'y activer.

La réputation de la Fédération CGT décourage les contempteurs du texte de 1947. Les hésitations des employeurs et des hauts fonctionnaires acquis de longue date au principe d'une réforme explosive ont cependant plusieurs causes. Favorable au desserrement de l'emprise cégétiste, une partie du patronat de la manutention ne se retrouve pas dans les projets «modernisateurs » de l'UNIM qui font la part trop belle aux grandes entreprises. Dans les hautes sphères administratives, plus d'un ingénieur des Ponts juge «chimérique »une «permanentisation » totale des dockers en dehors des quatre premiers ports français et des rares autres où une seule société intervient. ${ }^{28}$

Hormis le maillon faible phocéen, les syndicats ont su gérer, jusque-là, les évolutions inéluctables des professions portuaires. A Dunkerque, en 1962, les cégétistes arrachent une convention applicable au nouveau bassin minéralier utilisé par Usinor. ${ }^{29}$ Contre la garantie d'embauche exclusive d'une quinzaine de dockers professionnels rattachés au BCMO, le syndicat accepte des horaires inhabituels et, surtout, consent à la semi-permanentisation des ouvriers recrutés par roulement pour des périodes de seize semaines. Sur le moment, le compromis ne suscite pas de remontrances fédérales. Les militants, convaincus d'avoir sauvé l'essentiel, réussissent avec succès, en 1965, 1968 et 1969, à élargir la brèche ouverte. Tel est, de l'avis même d'Usinor, le prix à payer «pour s'insérer dans l'environnement local ». ${ }^{30}$

Sur un autre registre, les dockers havrais allient pareillement détermination stratégique et souplesse tactique. Informé de ce que leur port sera exposé à l'arrivée des conteneurs en provenance des Etats-Unis, leur secrétaire fait jouer ses relations américaines pour se procurer

\footnotetext{
${ }^{26}$ M. Pigenet, «Les dockers; retour sur le long processus de construction d'une identité collective en France, XIXe-XXe siècles », Genèses, n 42, mars 2001, pp. 5-25.

${ }^{27}$ M. Pigenet, «Le statut des dockers de 1947 : acquis législatif et pratiques sociales », in Actes du colloque

"Construction d'une histoire du droit du travail", Aix-en-Provence, 20-21 septembre 2000, Cahiers de l'Institut régional du travail, $\mathrm{n}^{\circ}$ spécial, pp. 241-259.

${ }^{28}$ Note du Service central des pmvn au ministre de l'Equipement, le 29 octobre 1969. CAC, 870150, art. 43.

${ }^{29}$ Elle s'inspire de l'accord conclu quelques mois plus tôt par le syndicat de Boulogne-sur-Mer et la Société Paris-Outreau qui traite les minerais de manganèse.

${ }^{30}$ Livre blanc de la Société Usinor sur le conflit qui paralyse le port de Dunkerque depuis le 10 mars 1977, le 26 avril 1977. CAC, 870150 art. 180.
} 
dans le plus grand secret la traduction de l'accord pionnier en vigueur à New York. ${ }^{31}$ Il en adapte les clauses au contexte hexagonal avant de présenter à la surprise générale un projet de taxation des conteneurs dont le produit alimentera une caisse de prévoyance en compensation de la diminution de la taille des équipes.

C'est à la Fédération que revient, en revanche, la défense du «statut», principal facteur de solidarisation nationale des dockers. Aussi bien y débat-on longuement des «permanents d'entreprise » et des «occasionnels» dont l'augmentation concurrence l'embauche des dockers professionnels intermittents, amenuise les recettes de la Cainagod et menace, à terme, l'indemnisation des vacations chômées. "Camarades dockers, attention! Les patrons du port veulent te faire disparaître (...), ils font rentrer sur le port des garçons qu'ils appellent pointeurs, conducteurs d'engins, etc. », alerte l'organe fédéral dès $1960 .{ }^{32} \mathrm{En}$ foi de quoi, les cégétistes tiennent les conducteurs d'engins pour des dockers et exigent qu'ils soient choisis exclusivement parmi les détenteurs de la carte professionnelle. ${ }^{33} \mathrm{Si}$ les dirigeants fédéraux concèdent qu'il serait «utopique» de prétendre contraindre les permanents à revenir dans les halls d'embauche des BCMO, ils prônent une stabilisation de leurs effectifs et la restitution de leur carte professionnelle en cas de licenciement. ${ }^{34}$

Sur ce point encore, les cégétistes marseillais se distinguent. Alors que les intermittents forment $35 \%$ des agents de maîtrise, les syndicalistes phocéens annoncent leur intention de porter leur part au-dessous de $15 \%{ }^{35}$ L'option va de pair avec un réaménagement des structures syndicales, source d'un nouveau contentieux avec la Fédération. La création de syndicats de spécialistes, tolérée pour les agents de maîtrise, provoque une levée de bouclier lorsque, à partir de 1962, plusieurs sections techniques du syndicat général accèdent au rang d'organisations à part entière, à l'exemple des primeuristes, des transitaires, des portefaix ou des porteurs de bagages. Les critiques se focalisent sur les conducteurs d'engins. En 1966, la progression du nombre des adhérents parmi les primeuristes - 240 contre 112 quatre ans plus tôt - ou les conducteurs d'engins - 400 sur 502 - ne nuit pas, plaide-t-on du côté de la Joliette, au syndicat général, passé de 714 membres à $1450 .{ }^{36}$ Ces initiatives, défendues au nom de la «décentralisation » préconisée par la Confédération, ${ }^{37}$ heurtent la majorité fédérale qui, inquiète d'un retour à l'éparpillement d'antan, condamne l'inscription du clivage intermittents/permanents dans les structures syndicales. Contre l'avis confédéral, la Fédération tient bon et refuser d'affilier le syndicat catégoriel litigieux.

A plus forte raison veille-t-elle à maintenir les « occasionnels» hors des syndicats. Confrontés à la présence massive et quotidienne de «complémentaires » embauchés dans les bars du port, les cégétistes marseillais sont à deux doigts de braver l'interdit. ${ }^{38}$ En 1962, une section aurait commencé à les réunir, mais les militants s'inclinent, cette fois, devant l'argumentaire fédéral fondé sur la protection du système institué en 1947. Depuis novembre

\footnotetext{
${ }^{31}$ Entretien avec Lucien Nolent, le 8 juin 2000 ; L'Avenir des Ports, décembre 1966.

${ }^{32}$ L'Avenir des Ports, juillet-août 1960.

${ }^{33}$ Intervention d'Henry, Congrès fédéral national, Paris, 13-14 juin 1962, p. 11.

${ }^{34}$ Intervention d'Henry, Congrès fédéral national, Paris, 18-19 juin 1970, p. 56. « Le plus sorcier d'entre nous ne réussirait pas à ce que les permanents acceptent de redevenir intermittents », confirme Gouvart quatre ans plus tard, Congrès fédéral national, Paris, 13-14 juin 1974, p. 48.

35 Avec des mensuels issus exclusivement des rangs des dockers intermittents, est-il toutefois précisé. Intervention de Reginensi, Congrès fédéral national, Paris, 22-23 juin 1966, p. 35.

${ }^{36}$ Interventions de Pacini et Gazelle, Idem, p. 68-69 et 31.

${ }^{37}$ Mal informé des tenants et des aboutissants corporatifs du débat, le représentant de la Confédération est vertement remis en place par Désiré Brest pour avoir réitéré les positions de la CGT en matière de décentralisation des syndicats. «Cela ne vous regarde pas (...). Ce n'est pas la peine de tenir un congrès si, après une décision du congrès, on doit combattre sur la même question devant la Confédération ». Intervention de Brest, Congrès fédéral national, Paris, 22-23 juin 1966, p. 88.

${ }^{38}$ Intervention de Manunta, Congrès fédéral national, Paris, 13-14 juin 1962, p. 42 et 96.
} 
1948, divers projets ont voulu le réformer. Tous visent, sans succès, la «rigidité » des procédures d'embauche, le nombre «excessif» de vacations chômées et de dockers professionnels.

\section{Perspectives libérales et nouvelles responsabilités patronales; la loi de 1947 en question}

Les mutations techniques raffermissent la détermination du patronat et des hauts fonctionnaires à sortir de l'intermittence les travailleurs les plus qualifiés, étape d'un double processus de stabilisation et de subordination. En janvier 1965, une commission spéciale de l'UNIM reprend le dossier. Ses conclusions, présentées en décembre de l'année suivante, tendent à acclimater en France les solutions en vigueur aux Pays-Bas et en Allemagne. Elles préconisent des mesures immédiates de renforcement des prérogatives patronales, jalons vers l'objectif de mensualisation du gros des ouvriers et d'affectation des derniers intermittents à des «groupements » interentreprises qui en mutualiseraient l'usage sur la base d'une garantie d'emploi et de ressources minimales. L'UNIM se sait en phase avec les autorités qui ont leur mot à dire sur une activité stratégique tenue pour « un des éléments du service public ». ${ }^{39}$

Aux yeux des pouvoirs publics, les ports constituent toujours un «maillon » essentiel du programme de modernisation et d'industrialisation arrêté par les $4^{\mathrm{e}}$ et $5^{\mathrm{e}}$ plans dans la perspective de l'intégration économique européenne et d'ouverture sur les marchés mondiaux. Le financement public prévu par le $5^{\mathrm{e}}$ Plan (1966-1970) va de pair avec la concentration de l'effort sur les principaux ports et l'implication accrue des acteurs privés. ${ }^{40}$ La loi du 29 juin 1965 institue à cet effet un régime d'autonomie pour les six premiers sites métropolitains. ${ }^{41}$ Il s'agit de "créer un cadre normal pour l'action des professions commerciales et industrielles dans une économie de marché $\gg .{ }^{42}$ Une étude commandée par le ministère des Finances sur les moyens de comprimer les coûts des opérations de manutention pour les soumettre «aux exigences de la compétition internationale », brosse en septembre 1966 un tableau complet de la situation. ${ }^{43}$ Au chapitre des propositions concernant la main d'oeuvre, il pointe sans surprise la "rigidité "présidant à son emploi, "l'anarchie des rémunérations », «l'inadaptation de la protection sociale », l'absence de règlements intérieurs aux entreprises, le maintien en activité de travailleurs inaptes... Le document ne voit pas là l'unique cause de la cherté relative des ports français et des « détournements de trafic » au bénéfice d'Anvers et de Rotterdam, mais n'érige pas moins la révision du «statut» de 1947 au nombre des solutions « souhaitables ». ${ }^{4}$

Cette convergence sur l'essentiel des propositions patronales et administratives ne doit rien au hasard. Les archives conservent maintes traces de l'étroite concertation entre les responsables patronaux et les ingénieurs des Ponts et Chaussées du ministère de l'Equipement. Plus que l'accord sur le fond, la nouveauté réside dans l'effort de concertation tactique. La direction des ports maritimes relève ainsi, en juillet 1967, les « délicats problèmes » posés par l'application du plan de l'UNIM. ${ }^{45}$ Face à «l'opposition attendue des

\footnotetext{
${ }^{39}$ Décisions du Conseil d'Etat des 23 juin 1939 et 5 mai 1944.

${ }^{40}$ Objectifs explicités dans le rapport Cordesse présenté devant le Conseil économique et social le 27 mars 1968. Journal de la marine marchande du 4 avril 1968.

${ }^{41}$ Le Havre et Bordeaux, Marseille, Dunkerque, Nantes-Saint-Nazaire et Rouen.

${ }^{42}$ Note interne de la direction des pmvn, le 12 janvier 1971. CAC, 870150, art. 161.

${ }^{43}$ D'abord confiée à M. Gavois, elle revient bientôt à J. Choussat, également inspecteur des Finances, qui signe le rapport final auquel son nom sera désormais associé. Journal de la Marine marchande du 22 septembre 1966.

${ }^{44}$ Le rapport préconise également la libération des tarifs jusque-là administrés et la redistribution des frais de manutention entre les différents acteurs portuaires.

${ }^{45}$ Note de J. Chapon sur le projet de réforme de la loi de 1947 par l'UNIM, le 19 juillet 1967. CAC, 870150, art. 12.
} 
syndicats », ${ }^{46}$ elle recommande d'avertir le Premier Ministre du risque d' «incidents sociaux prévisibles ». Au nombre des «conditions favorables » au succès de la réforme, la note parie sur l'incompréhension du reste "monde ouvrier», alors préoccupé par la dégradation de l'emploi, devant l'opposition des dockers à une mensualisation censée être plus protectrice que l'intermittence. Si le cabinet du chef du gouvernement donne son feu vert à la réforme, il écarte l'idée d'un projet de loi prématuré, lui préférant la voie progressive de mesures réglementaires destinées à saper les fondations du système en place. ${ }^{47}$ Dans l'immédiat, Matignon exige d'être informé de l'avancement d'une affaire reconnue importante. On est en mars $1968 \ldots{ }^{48}$ Les grèves du printemps torpillent l'agenda du gouvernement peu désireux d'alourdir davantage le contentieux social.

\section{Conclusion : retour sur les années 1968 dans les ports}

$\mathrm{Au}$ sortir des événements, les débats et la réorganisation qui agitent son sommet fragilisent la Fédération CGT dont la nouvelle direction doit trouver ses marques. Affaiblie sur le front social, mais exempte de contestation interne, l'UNIM teste ses interlocuteurs. Mal lui en prend. Coup sur coup, plusieurs conflits d'envergure remettent les ports sur le devant d'une scène sociale alors très investie et ressoudent les militants. Sur les quais aussi, l'expérience de 1968 attise la combativité, notamment des jeunes dont une enquête de 1971 signale la radicalisation à Marseille. ${ }^{49}$

Après soixante-dix sept jours de grève, la Fédération vient à bout, le 2 décembre 1969, de la dénonciation par les employeurs des clauses de l'accord Marceau sur la réduction du temps de travail et le maintien d'un rapport constant entre le SMIG et le salaire de base. La lutte fait également capoter les propositions visant à «combler les lacunes » de la loi de 1947 dans le sens d'un renforcement de l'autorité patronale. Elle reprend, cependant, pour neuf mois en septembre 1970. Cette fois, le différend mêle l'opposition à un projet gouvernemental inspiré des anciennes propositions de $1^{\prime} \mathrm{UNIM}^{50}$, le combat contre la «privatisation des quais » qui se profile à Usinor-Dunkerque ${ }^{51}$ et un ensemble de revendications allant de l'augmentation des salaires et du doublement de l'indemnité versées pour les vacations chômées au départ anticipé des dockers de 60 ans pour cause d'inaptitude au travail et à l'amélioration de la protection sociale. Au printemps 1971, le ministre de l'Equipement Albin Chalandon - suggère une issue de type «donnant-donnant ». ${ }^{52}$ En contrepartie de la satisfaction des revendications ouvrières, engagement compris de favoriser l'emploi des

\footnotetext{
${ }^{46}$ Les dockers «n'accepteront jamais de devenir des esclaves » prévient la Fédération. La Vie ouvrière du 31 mars 1967.

${ }^{47}$ Note de J. Chapon du 18 mars 1968. CAC, 870150, art. 12.

${ }^{48}$ Le 27 mars 1968, le rapport Cordesse soumis à l'avis du Conseil économique et social préconise à son tour une modification profonde de la situation des dockers. Journal de la marine marchande du 4 avril 1968.

${ }^{49}$ L'enquête de la SOFRES réalisée parmi les dockers de ce port en 1971 conclut sur l' « excellente image » de la CGT perçue comme l'organisation « qui défend le mieux les dockers ». La radicalisation ne suscite pas moins, selon les enquêteurs, « une divergence croissante » entre jeunes et vieux dockers à propos des moyens de lutte. Les dockers de Marseille, mai 1971. CAC, 870150, art. 194.

50 «Nous sommes bien d'accord sur l'objectif poursuivi », écrit le directeur des pmvn au responsable de l'UNIM, le 22 septembre 1970, non sans rappeler les deux conditions à l'amélioration de la compétitivité des ports : le progrès social et le renforcement de l'autorité des employeurs sur leurs salariés. CAC, 870150, art. 12.

${ }^{51}$ L'accord dunkerquois du 15 janvier 1969 conforte l'emploi de dockers semi-permanents, acquis depuis 1962 , mais l'entreprise, irritée par la multiplication des actions revendicatives envisage de les remplacer par son propre personnel. La Fédération souhaite d'autant plus donner un coup d'arrêt à ces prétentions que la question se posera bientôt sur le site en construction de Fos. M. Pigenet, « Normes juridiques... », art. cit.

52 Dans le huis-clos du Conseil des ministres réuni le 16 juin 1971, A. Chalandon explique qu'il a aggravé volontairement le conflit pour contrebalancer la faiblesse des employeurs et contraindre les dockers à des contreparties. Communication au Conseil des ministres. CAC, 870 150, art. 164.
} 
dockers professionnels sur les terminaux concédés aux entreprises industrielles, des accords locaux réduiraient la composition des équipes pour tenir compte des progrès techniques et mettraient fin aux «pratiques restrictives » des dockers. Le 4 juin 1971, un accord national consigne le versant paritaire du compromis, tandis que la reprise générale du travail coïncide, le 14 , avec la signature d'une circulaire qui officialise les promesses du ministre. ${ }^{53}$ Le problème du recours aux dockers pour le déchargement des minerais rebondit néanmoins à Fos-sur-Mer en octobre 1973, conduisant à une nouvelle victoire ouvrière que scelle la convention du 22 juillet 1974. La même année, l'accord national du 17 octobre amène l'UNIM à accepter que les dockers permanents soient recrutés exclusivement parmi les professionnels intermittents. ${ }^{54}$ Le patronat renonce, en outre, à conclure des accords d'entreprises et consent à ce que les ports demeurent le cadre exclusif des conventions collectives. ${ }^{55}$ Aux dires d'un dirigeant de l'UNIM, le texte « consacre l'intermittence ». ${ }^{56}$ Il consolide le « curieux régime de libre entreprise avec main œuvre obligatoire ${ }^{57}$ parfaitement maîtrisé par les syndicats dont la pression concourt au relèvement des gains des dockers qui, bientôt supérieurs aux salaires moyens des ouvriers hexagonaux ${ }^{58}$, se rapprochent de ceux de leurs homologues belges ou néerlandais.

Déjà, des militants imaginent de nouvelles conquêtes par extension de la sphère d'intervention des dockers. A Dunkerque, le syndicat ouvre ses rangs à l'ensemble des travailleurs du site: contremaîtres, conducteurs d'engins, pointeurs, menuisiers, gréeurs, magasiniers, mécaniciens et... portiqueurs d'Usinor. ${ }^{59} \mathrm{Si}$ tous ne sont pas encore détenteurs d'une carte professionnelle, les cégétistes préparent les conditions pour qu'à terme tous les emplois soient accessibles aux dockers. "Nous tenons l'avenir en tenant tous les travaux », résume Roger Gouvart. ${ }^{60}$ Sur la lancée, il invite à reconsidérer «notre conception de la mission des Ports et Docks » et à solidariser «toutes nos ramifications qui s'étalent sur le pays : les ports, les fleuves, les canaux, les quais fluviaux, les docks, les aéroports... ». A Fos et à Marseille, des militants songent pour leur part à implanter le syndicat dans la « deuxième zone » des « ports secs » où s'effectue le dépotage des conteneurs. ${ }^{61}$

Réponses au recul des emplois traditionnels ${ }^{62}$ à la suite de spectaculaires gains de productivité, ces objectifs butent sur la trop rapide augmentation des vacations chômées qui bouleverse le rapport des forces sociales. En 1979, plusieurs textes réglementaires réactivent la remise en cause de la loi de 1947. La cotisation patronale uniforme à la Cainagod est ainsi

\footnotetext{
${ }^{53}$ Notons qu'en avril, un arrêté destiné à encourager les employeurs à recruter des dockers permanents réduit le taux de cotisation sur ces emplois. Un second définit plus précisément les règles d'attribution et de retrait des cartes professionnelles.

${ }^{54}$ Acceptation quelque peu contrainte : deux jours avant cette signature, le président réaffirmait l'opposition des employeurs à la généralisation de l'intermittence qui «mettrait leur activité à la discrétion entière de la Fédération des Ports et Docks ». Note du 15 octobre 1974 au Directeur général de la concurrence et des prix. CAC, 870150, art. 106.

${ }^{55}$ Favorable à la négociation d'accords partiels, la Fédération refuse de débattre d'une convention collective nationale dont elle craint qu'elle ne vienne menacer le «statut» de 1947.

${ }^{56}$ L. Hislaire, Dockers, corporatisme et changement, Paris, Transport Actualités-GEP Communication, 1993, p. 63.

${ }^{57}$ Selon la formule d'une note de la direction des pmvn, le 27 juin 1979. CAC, 870150, art. 168.

58 En 1970, le salaire moyen mensuel d'un docker professionnel intermittent - 16927 francs - est encore inférieur à la moyenne de l'ensemble des ouvriers - 20277 francs. Le dépassement interviendra vers 1975. Statistiques du service central de la direction des pmvn, le 17 novembre 1970. CAC, 870150, art. 161.

${ }^{59}$ Intervention de Gouvart, Congrès fédéral national, Paris, 13-14 juin 1974, p. 48.

${ }^{60}$ Intervention de Gouvart, Congrès fédéral national, Paris, 11-12 juin 1976, p. 60-61. En 1977, Usinor perd en avril la nouvelle épreuve de force engagée à propos du futur et coûteux quai aux aciers, bientôt revu à la baisse pour cause de crise sidérurgique.

${ }^{61}$ Intervention de Bergeneau, Congrès fédéral national, Paris, 8-9 juin 1978, p. 17.

${ }^{62}$ On dénombrait près de 22000 dockers professionnels intermittents en 1947, 18280 en 1953, 15945 en 1965 , à 10694 en 1970. Ils tomberont à 8800 en 1989.
} 
remplacée par un taux modulable au gré des situations locales, altération significative du dispositif de péréquation nationale établie à la Libération. Les hauts fonctionnaires ne cachent pas leur intention d'instituer une situation irréversible «d'autonomie et (de) responsabilité des employeurs et des dockers au niveau de chaque port ${ }^{63}$ Pas à pas, si possible, mais sans exclure une sévère riposte judiciaire en cas de conflit. ${ }^{64}$

Une nouvelle période commence, celle de la déréglementation du travail portuaire dont le caractère mondial ne laisse guère de doute sur la défaite inéluctable de la résistance ouvrière hexagonale qui, prolongée jusqu'en 1992, permettra néanmoins de la monnayer plus chèrement qu'ailleurs. ${ }^{65}$

\footnotetext{
${ }^{63}$ Direction des pmvn, note du 27 juin 1979. CAC, 870150, art. 168.

${ }^{64}$ Service central d'exploitation. Réflexions sur la stratégie à suivre en cas d'éventuel conflit avec les dockers en novembre 1979, le 24 septembre 1979. CAC, 870150, art. 168.

${ }^{65} \mathrm{M}$. Pigenet, «Les origines d'une configuration sociale singulière : logiques et acteurs des dynamiques sociales portuaires en Europe au XXe siècle ; une approche comparative » in J.-P. Le Crom (dir.), Les acteurs du droit du travail, Rennes, PUR, 2004, p. 371-381.
} 